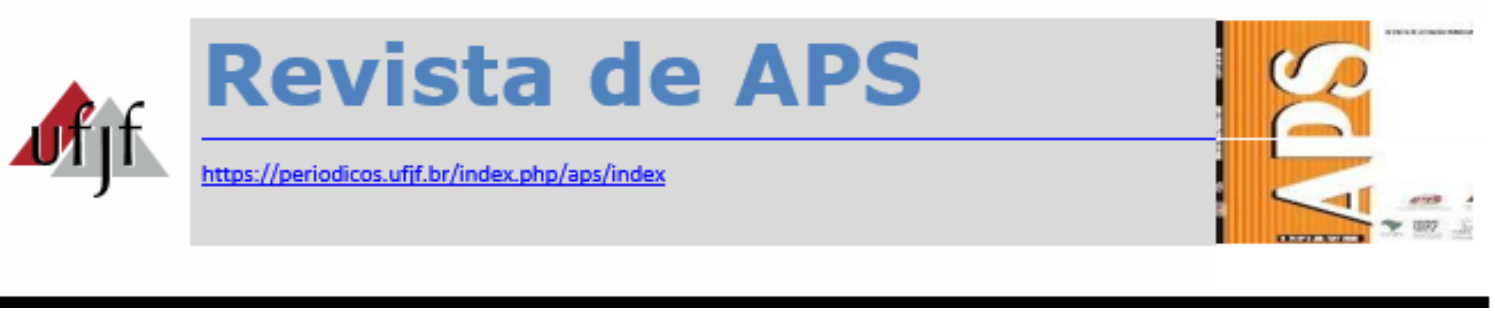

\title{
Esporotricose brasileira: desdobramentos de uma epidemia negligenciada
}

\section{Brazilian sporotrichosis: development of a neglected epidemic}

\author{
Rebeca Mól Lima ${ }^{1}$, William Lucas Ferreira da Silva ${ }^{2}$, Jordana de Abreu Lazzarini ${ }^{3}$, \\ Nádia Rezende Barbosa Raposo ${ }^{4}$
}

\section{RESUMO}

A esporotricose é uma micose subcutânea de distribuição mundial, sendo observada em surtos de proporções variáveis, com predominância em áreas de clima temperado e tropical. Algumas áreas de endemicidade já foram relatadas. No Brasil, seus agentes etiológicos encontraram não só condições climáticas e estruturais ideais, mas também fontes de infecções extremamente eficientes. Nas últimas décadas, o país tem observado um aumento exponencial no número de casos de esporotricose humana e animal. Esse aumento e o surgimento de resistência aos antifúngicos tornam a situação alarmante. Apesar de essa epidemia se perpetuar há anos, existem poucas políticas de saúde no Brasil para o controle dos casos de esporotricose humana e animal, principalmente na atenção primária à saúde. Assim, intervenções sociais, ambientais e populacionais devem ser feitas para conter essa epidemia negligenciada.

PALAVRAS-CHAVE: Esporotricose. Epidemia. Sporothrix schenckii. Políticas de saúde.

\section{ABSTRACT}

Sporotrichosis is a subcutaneous mycosis with a worldwide distribution, which has been observed in outbreaks of variable proportions in all continents, with predominance in temperate and tropical climate zones. Some areas of endemicity

\footnotetext{
${ }^{1}$ Farmacêutica. Mestra em Saúde. Núcleo de Pesquisa e Inovação em Ciências da Saúde da Universidade Federal de Juiz de Fora (Nupics/UFJF).

${ }^{2}$ Farmacêutico. Especialista em Farmácia Clínica e Hospitalar. Mestrando em Saúde Coletiva. Núcleo de Pesquisa e Inovação em Ciências da Saúde da Universidade Federal de Juiz de Fora (Nupics/UFJF). E-mail: williamluccas@yahoo.com.br.

${ }^{3}$ Farmacêutica. Mestra em Ciências Farmacêuticas. Núcleo de Pesquisa e Inovação em Ciências da Saúde da Universidade Federal de Juiz de Fora (Nupics/UFJF).

4 Farmacêutica. Doutora em Toxicologia. Professora do Departamento de Ciências Farmacêuticas da Universidade Federal de Juiz de Fora. Núcleo de Pesquisa e Inovação em Ciências da Saúde (Nupics/UFJF).
} 
have already been reported. In Brazil, its etiological agents found not only ideal climatic and structural conditions, but also extremely efficient sources of infections. In the last decades, the country has suffered an exponential increase in the number of human and animal cases of sporotrichosis. This increase, combined with the emergence of antifungal resistance makes the situation alarming. Although this epidemic has been perpetuating for years, there are few health policies in Brazil to control it/the disease, especially in primary health care. Therefore, social, environmental and population-based interventions should be organized in order to contain this neglected epidemic.

KEYWORDS: Sporotrichosis. Epidemy. Sporothrix schenckii. Health policies.

\section{INTRODUÇÃO}

A esporotricose é uma micose subcutânea que se caracteriza pelo aparecimento de lesões nodulares dos tecidos cutâneos e subcutâneos, com supuração e ulceração, ocasionada por espécies de fungos dimórficos e geofílicos do complexo Sporothrix schenckii. ${ }^{1-3} \mathrm{~A}$ doença tem evolução subaguda a crônica e acomete seres humanos e animais, como cães, gatos, roedores, tatus, cavalos, bovinos, macacos e aves. ${ }^{4,5}$ As formas clínicas mais comuns são a cutânea fixa e a linfocutânea, mas outras manifestações também são relatadas, como a extracutânea e a sistêmica. ${ }^{5-7}$

Por muitos anos, pensou-se que a esporotricose era causada por um agente apenas, porém estudos moleculares com diferentes amostras de S. schenckii relatam a existência de variações fenotípicas entre as linhagens fúngicas caracterizando um complexo. ${ }^{1,8,9}$ Tais variações compreendem diferenças morfológicas e crescimento em meios de culturas distintos, ${ }^{10,11}$ na termotolerância, ${ }^{12,13}$ na secreção de proteínas, ${ }^{14}$ na síntese de melanina, ${ }^{15,16}$ na virulência em modelo murino ${ }^{1,17-19}$ e na suscetibilidade aos agentes antifúngicos. ${ }^{20}$

Seus agentes etiológicos são fungos dimórficos e termotolerantes do complexo S. schenckii, os quais podem ser encontrados na forma filamentosa dispersos no solo, na vegetação, na água, na matéria orgânica ou na forma leveduriforme como parasita. ${ }^{1,4,9}$ Neste complexo, quatro espécies de relevância clínica são descritas: $S$. schenckii sensu stricto (clado II), S. brasiliensis (clado I), S. globosa (clado III) e S. Iuriei (clado IV). ${ }^{8,9,20,21}$ Além das diferenças fenotípicas e genotípicas, apresentam distribuições geográficas distintas. ${ }^{7,22}$

A esporotricose possui distribuição mundial, ocorrendo tanto em área rural quanto urbana, com prevalência em regiões de clima temperado e tropical. Há relatos 
de endemicidade na África do Sul, na Índia, na China, no Japão, no Peru, na Colômbia e no Brasil. ${ }^{9,16}$ Essa doença também está associada a casos clínicos esporádicos em países como Itália, ${ }^{23}$ Portugal ${ }^{24}$ e Grécia. ${ }^{25}$ No entanto, descreve-se a maioria dos casos nas Américas Central e do Sul. ${ }^{9,16}$

A forma de transmissão clássica está ligada à inoculação traumática do fungo por uma fonte infectante, como farpas e espinhos. Contudo, nas últimas décadas, relatos de transmissão zoonótica, por arranhaduras ou mordeduras de animais contaminados, vêm mudando esse panorama..$^{1,4,9}$

A esporotricose apresenta diferentes formas clínicas de acordo com a virulência do patógeno, da condição imunológica do hospedeiro e da rota de infecção. São descritas as formas cutâneas (cutânea fixa, linfocutânea e cutânea disseminada) e extracutâneas (pulmonar primária, ocular, articular e ósseo) e a sistêmica. As formas clínicas mais comuns são a cutânea fixa e a linfocutânea. ${ }^{6-8}$

Por muito tempo, o tratamento da esporotricose baseou-se na ingestão de soluções de iodo. No entanto, sua alta toxicidade impulsionou seu progressivo desuso. ${ }^{26}$ Atualmente, o itraconazol, o iodeto de potássio, a anfotericina $B$ e a terbinafina figuram como os mais extensivamente utilizados. ${ }^{19,26,27}$ A anfotericina B é recomendada para casos sistêmicos ou na impossibilidade de utilização do itraconazol, como no caso de mulheres grávidas. ${ }^{26,27} \mathrm{Em}$ todos os casos, o tratamento mostra-se demorado, durando de três a seis meses nas formas mais restritas da doença. Na forma disseminada ou em pacientes imunocomprometidos, pode haver o prolongamento do uso dos fármacos. ${ }^{19,26-28}$

No diagnóstico da esporotricose, a suspeita clínica mostra-se essencial. Deve-se aliar uma anamnese detalhada com dados epidemiológicos, manifestações clínicas e exames complementares. ${ }^{29,30}$ A confirmação definitiva só é obtida através do cultivo de amostras com sucessivo isolamento e identificação do fungo. $O$ padrão-ouro de diagnóstico da esporotricose é a cultura, mas métodos sorológicos, histopatológicos e moleculares têm sido recentemente adotados como ferramentas auxiliares na detecção. ${ }^{6,29-31}$

A esporotricose não é uma doença de notificação compulsória, com exceção em alguns municípios. Dessa maneira, sua prevalência ainda se mostra obscura. Os dados epidemiológicos conhecidos provêm, em sua maior parte, de artigos científicos 
publicados e de levantamentos de órgãos de saúde pública, os quais apontam o aumento da incidência na última década. ${ }^{12,26,30}$

Diante do aumento da incidência, a esporotricose tem se tornado um agravo de relevante interesse em saúde pública. Nesse sentido, o presente estudo teve como objetivo descrever as epidemiologias brasileira e mundial, bem como discutir as políticas públicas de saúde e o papel da atenção primária à saúde no contexto.

\section{METODOLOGIA}

A revisão bibliográfica acerca do tema epidemiologia da esporotricose foi estabelecida por meio de pesquisas em documentos do Ministério da Saúde, da Organização Mundial da Saúde e das bases de dados Scientific Eletronic Library Online (SciELO), Medline/Pubmed, PubMed Central (PMC), The Jama Network Journals of the American Medical Association e Biblioteca Virtual em Saúde Brasil (BVS Brasil), utilizando-se os seguintes descritores certificados pelos Descritores em Ciências da Saúde (DeCS): esporotricose, epidemia, Sporothrix schenckii, Políticas de saúde, Sporotrichosis, Epidemy, Sporothrix schenckii e Health policies, além da literatura.

As publicações utilizadas na elaboração deste trabalho foram selecionadas com base nos seguintes critérios de seleção: artigos publicados até o ano de 2018, nos idiomas inglês e português, que apresentassem informações e dados sobre a epidemiologia de esporotricose e as políticas de saúde brasileira. Excluíram-se as publicações que não contemplavam o objetivo proposto, bem como artigos sem metodologia descrita. Da posse de todos os dados, foi redigida esta revisão.

\section{DESENVOLVIMENTO}

\section{Epidemiologia mundial}

No cenário mundial, a esporotricose foi primeiramente descrita pelo estudante de Medicina Benjamin Schenck, em 1896, no Hospital Johns Hopkins em Baltimore, no Estados Unidos, ao tratar de uma lesão de pele no membro superior direito de um paciente. O fungo isolado dessa lesão na mão e braço foi avaliado pelo micologista Erwin 
F. Smith e identificado como uma espécie pertencente ao gênero Sporotrichum. Essa denominação errônea permaneceu por décadas mesmo depois de Hektoen e Perkins, em 1900, proporem uma nova denominação. ${ }^{6,22}$

Segundo Chakrabarti et al. (2015), a esporotricose destaca-se entre as doenças causadas por fungos dimórficos, cuja endemicidade é globalmente distribuída, apresentando características ecológicas, epidemiológicas e clínicas variáveis de acordo com as diferentes regiões geográficas. Além disso, seus agentes etiológicos estão distribuídos mundialmente no ambiente, sendo a única doença causada por fungos dimórficos que tem transmissão zoonótica substancial. ${ }^{22}$

A esporotricose apresenta distribuição mundial, com maior prevalência em regiões de clima temperado e tropical, onde o fungo encontra as condições mais favoráveis para seu desenvolvimento. Tais áreas contemplam América Latina (Brasil, México, Colômbia, Costa Rica, Guatemala e Uruguai), África do Sul, Índia e Japão, sendo a maioria dos casos descrita nas Américas Central e América do Sul. ${ }^{21,22,32-34}$

A esporotricose não é de notificação compulsória em todos os países, fato este que dificulta a obtenção de dados e estabelecimento de suas incidência e prevalência ${ }^{6}$ Em algumas áreas, vem diminuindo o número de casos relatados, como na França e no Japão, ${ }^{35,36}$ que apresentavam elevada incidência no início do século e, atualmente, registram raros casos da doença. Outras registram um aumento nos últimos anos, como a Austrália e o Brasil. 21,22,32-38 Países como Peru e México já apresentam áreas hiperendêmicas com registros alarmantes de 48 a 98 casos por 100 mil habitantes e 25 casos por 1.000 habitantes, respectivamente..$^{39,40}$

A doença pode afetar qualquer pessoa, independentemente de sexo ou idade, e a proporção de homens para mulheres acometidos varia de região para região. Em alguns países, hábitos ocupacionais ou recreativos específicos daquela população podem aumentar o risco de contrair a doença, expondo seus habitantes a um maior contato com o fungo. ${ }^{6,22,41}$ Por exemplo, na África do Sul e no Uruguai, a esporotricose tem maior incidência em homens do que em mulheres. Este fato decorre da intensa atividade mineradora, na África do Sul, e da caça de tatus, no Uruguai - exercida majoritariamente por homens, os quais se expõem regularmente ao agente etiológico.9,42 Já na Índia e no Japão, as mulheres são as mais acometidas pela doença, por estarem envolvidas com atividades agrícolas. ${ }^{35,43}$ No entanto, em outras regiões do 
mundo, não foi possível estabelecer esse tipo de correlação. ${ }^{40}$

Por seu agente etiológico ser encontrado disperso no ambiente, a transmissão clássica da doença ocorre pela inoculação traumática do fungo por meio do contato com madeiras, vegetais e solo contaminado. Devido a esse fato, a esporotricose é tradicionalmente conhecida como "doença de jardineiro", por sua maior incidência em agricultores, floricultores, horticultores e fazendeiros. ${ }^{32}$ Alguns surtos de esporotricose foram relatados ao longo dos anos, como o surto envolvendo mais de 3.000 mineiros na África do Sul pela contaminação da madeira de suporte das minas, nos anos 1940, considerada a maior epidemia do século $\mathrm{XX} .{ }^{44}$

Contudo, um novo grupo de risco vem ganhando força nos últimos anos, decorrente da ampliação dos casos de transmissão zoonótica da doença por arranhadura e mordedura de animais. Os animais mais citados como responsáveis pela transmissão são gatos e tatus, mas existem relatos envolvendo roedores, cães, cavalos, aves e até insetos. ${ }^{6,32,45}$ Casos de transmissão zoonótica vêm sendo relatados na Argentina e no Uruguai (tatus) e, na Malásia e no Brasil (gatos). ${ }^{46-48}$

\section{Epidemiologia no Brasil}

A esporotricose foi primeiramente identificada em 1907 no país, quando relataram a infecção no homem e em animais (ratos) no estado de São Paulo. A partir daí, outros casos têm sido descritos, especialmente nos estados de São Paulo, Rio de Janeiro e Rio Grande do Sul, culminando com a recente caracterização epidemiológica na região do Rio de Janeiro. Esta envolve municípios vizinhos e é marcada pelo acometimento de felinos, homens e cães. ${ }^{7,32,49,50}$

Desde o fim da década de 1990, a doença vem ganhando caráter epidêmico no estado do Rio de Janeiro (região litorânea, quente e úmida), sendo um dos maiores surtos de esporotricose em humanos e animais já registrados. O aumento exponencial de casos da doença é observado no Sudeste, porém diversas regiões começaram a registrar casos nos últimos anos. ${ }^{51,52}$

Entre 1998 e 2004, a Fundação Oswaldo Cruz (Fiocruz) diagnosticou 1.503 gatos, 64 cachorros e 759 humanos com a doença, em contraste com apenas 13 casos em 1987 e $1998 .^{38}$ Dados mais recentes descrevem, aproximadamente, 2.200 casos da doença 
em humanos e 3.244 em gatos entre 1998 e 2009, caracterizando a maior epidemia (animal e humana) já registrada no mundo. $22,37,53$

Entre 2008 e 2011, mais 2.340 novos casos de esporotricose humana foram confirmados, de acordo com dados do Instituto de Pesquisas e Estudos Sociais (Ipes). ${ }^{56}$ Segundo o Boletim Epidemiológico da Gerência de Doenças Transmitidas por Vetores e Zoonoses da Secretaria de Estado de Saúde do Rio de Janeiro, no banco do Sistema Nacional de Agravos de Notificação do Estado do Rio de Janeiro (Sinan/RJ) durante os anos de 2016 e 2017, notificaram-se 1.485 e 1.741 casos de esporotricose, respectivamente. No somatório dos dois anos, 2.499 casos foram confirmados, sendo 75,7\%, em 2016, e 79\% em $2017 .{ }^{55}$ Os números de casos estão aumentando continuamente há mais de 17 anos e já somam mais de 5.000 registros no Instituto Nacional de Doenças Infecciosas Evandro Chagas (INI). ${ }^{51}$

Tal epidemia difere em sua forma de transmissão dos surtos relatados ao redor do mundo, já que a rota de infeç̧ão ocorre, predominantemente, pela inoculação traumática por arranhadura ou mordedura de gatos infectados. ${ }^{32,51}$ Os felinos, considerados como ponto crítico na ecoepidemiologia da esporotricose, tornaram-se fontes de infecção eficientes por seus hábitos de vida livre, saindo de sua casa para circular na vizinhança, caçar pássaros e ratos, se relacionar com outros gatos, esconder suas fezes no solo, arranhar árvores e se envolver em brigas, o que aumenta as chances de contato com o fungo. Além disso, estudos demonstraram a presença da forma leveduriforme em lesões, unhas e cavidade oral de felinos com esporotricose, facilitando a disseminação do microrganismo não só no ambiente, mas também entre os próprios gatos e humanos. ${ }^{54,56,57}$

Outro agravante é o tamanho da população de felinos no Brasil. Segundo a Pesquisa Nacional de Saúde do IBGE (2013), 44,3\% dos domicílios brasileiros possuem, pelo menos, um cachorro; e 17,7\% têm, pelo menos, um gato, o que demonstra um elevado percentual de animais de estimação. Esses dados contribuem para o desenvolvimento de novas políticas de saúde, desenvolvimento e programação de vacinas. ${ }^{58,59}$

Estudos demonstraram que a epidemia possui um perfil bem delineado, ocorrendo em áreas mais pobres da região metropolitana e arredores da cidade do Rio de Janeiro, e acomete predominantemente mulheres adultas, na faixa de 40 a 59 anos, 
fora do mercado de trabalho, as quais se dedicam a atividades domésticas, entre elas, 0 cuidado de animais domésticos. ${ }^{32,52,56,60}$ A Secretaria de Saúde do estado de São Paulo promoveu um estudo de identificação ativa dos casos da doença em humanos e felinos, visando a traçar o perfil epidemiológico. Neste estudo, 163 casos de esporotricose felina foram identificados ao longo de três anos, 2011 a 2013. Enquanto isso, os casos de esporotricose em humanos somaram apenas 11 casos, de 2011 a 2012. ${ }^{51}$

No estado do Espírito Santo, os casos são descritos em regiões onde a altitude e as temperaturas são diferentes do Rio de Janeiro. No entanto, há prevalência da transmissão clássica, em que a inoculação traumática acidental acontece durante a manipulação de material vegetal contaminado, havendo poucos casos relacionados como zoonose..$^{58}$

No estado do Rio Grande do Sul, a caça de tatus é um mecanismo tanto de transmissão zoonótica quanto ocupacional. Duas cidades concentraram casos de esporotricose de transmissão zoonótica: Pelotas, que registrou seis casos em humanos, sendo dois destes relacionados aos donos de animais e um atendente de clínica veterinária; e Rio Grande, que registrou 64 felinos confirmados com esporotricose entre junho de 2010 e junho de $2013 .^{51}$

Gutierrez-Galhard et al. (2015) ressaltam que, apesar de não existirem muitos estudos descritos em outros estados do país, são registrados casos. Portanto, indícios da presença da esporotricose podem ser encontrados no Amazonas, no Pará, no Mato Grosso, em Goiás, no Piauí, no Ceará, na Paraíba, em Pernambuco e no Paraná. ${ }^{51}$

\section{Políticas de Saúde no Brasil}

Bustamante e Campos (2004) denominavam a esporotricose como uma doença esquecida das agendas, por ocorrer em áreas geográficas restritas e em desenvolvimento. ${ }^{56}$ Já em 2012, Silva et al. classificaram a esporotricose como uma doença negligenciada, que se caracteriza pela prevalência em áreas mais pobres, onde a população não representa compradores em potencial para indústrias farmacêuticas investirem em pesquisa. ${ }^{52}$

Esse descaso não se faz apenas por parte das empresas, mas também pelos governos. As medidas intervencionais de saúde para a prevenção e/ou a erradicação da 
doença acontecem de forma lenta, assumindo distintas características de acordo com a localidade acometida. Contudo, é unânime a importância do papel dos profissionais de saúde para que as medidas sejam eficientes, principalmente na educação em saúde junto à população. Ainda assim, o atendimento e o diagnóstico dos pacientes são precários, e não existe controle das principais formas de transmissão. Até dados sobre a doença são difíceis de serem obtidos, pois ela não é de notificação obrigatória na maioria dos estados brasileiros. ${ }^{32,37}$

Nesse contexto, a criação de políticas de saúde para o combate à esporotricose se torna imprescindível para frear e controlar a epidemia no Brasil. A Secretaria de Saúde do Estado (SES) do Rio de Janeiro deu o primeiro passo para o mapeamento da doença criando a Resolução SES no 674 de 12/07/2013 e aprovando o Informe Técnico 005 de 2014. Essa nova política redefine a relação de doenças e agravos de notificação compulsória no âmbito do estado do Rio de Janeiro e inclui a esporotricose como de notificação obrigatória através do Boletim Individual de Notificação, no Sistema de Informação de Agravos de Notificação (Sinan), tanto em casos de suspeita quanto de confirmação. ${ }^{62,63}$

Outras localidades estão aderindo à medida de notificação compulsória, que é importante para elucidar o perfil epidemiológico da doença e possibilitar futuras ações mais direcionadas ao impacto da doença no âmbito individual e coletivo, caso do município de Salvador (Bahia) que aprovou a nota técnica da Diretoria de Vigilância em Saúde no 04 de 23 de fevereiro de 2018. Esta também recomenda a notificação e o registro dos casos de esporotricose no Sinan. ${ }^{64}$

Barros et al. (2010) evidenciam a falta de um programa de saúde pública para o controle da esporotricose e sugerem estratégias para seu combate, como distribuição gratuita dos medicamentos, capacitação dos profissionais de saúde, ações de controle para os animais e ampla divulgação de informações sobre a doença. ${ }^{37} \mathrm{O}$ tratamento de escolha para as formas mais comuns da doença é o itraconazol, o qual pode estar disponível no componente básico da atenção primária à saúde de acordo com cada município, conforme a Relação Nacional de Medicamentos Essenciais de 2017 (Rename). Silva et al. (2012) destacam o alto custo mensal de itraconazol, de aproximadamente $\mathrm{R} \$ 160,00$ o medicamento genérico. Assim, o elevado custo do tratamento e sua longa duração dificultam a adesão pelo paciente, sobretudo quando 
há falta do medicamento nas unidades de saúde. ${ }^{65}$

A disponibilização desse medicamento para o tratamento dos casos confirmados evitaria a progressão da doença para formas mais agressivas capazes de levar os pacientes à morte, conforme observado em dois casos de indivíduos com síndrome de imunodeficiência adquirida (aids), e forneceria altas chances de cura para os pacientes, como demonstrado por Freitas et al. (2010). Segundo tais autores, a evolução para cura foi observada em $89,3 \%$ dos casos utilizando itraconazol no tratamento. ${ }^{66}$

O maior desafio da epidemia de esporotricose no Brasil ocorre pelo fato de esta apresentar transmissão zoonótica. Com os hábitos de vida livre dos felinos, a falta de controle da doença animal e a ausência de locais adequados para seu tratamento e seu diagnóstico, muito ainda precisa ser feito para frear essa disseminação.

Barros et al. (2010) também sugerem a criação de unidades de atendimento móvel para os animais doentes, fornecendo tratamento e castração dos felinos, eutanásia nos casos impossibilitados de terapêutica, cremação dos animais que evoluíram para óbito e educação para posse responsável de animais domésticos. Isso porque a taxa de abandono dos animais doentes é alta, contribuindo para a perpetuação do ciclo de transmissão do fungo. Dos pacientes com suspeita de esporotricose que procuraram a Fiocruz para um primeiro atendimento, cerca de $70 \%$ deles deu um destino inadequado a seu animal. ${ }^{32,67}$ Alguns autores explicam esse fato à dificuldade de transporte adequado do animal doente, além do alto custo e da longa duração do tratamento. ${ }^{37}$ Essas unidades diminuiriam efetivamente o contágio de humanos se garantissem não só o diagnóstico, mas também o tratamento gratuito, diminuindo o número de animais infectados.

Para o controle do ambiente utilizado por esses animais, torna-se imprescindível a adequação de infraestrutura e saneamento básico, a fim de diminuir a incidência de roedores, os quais também são infectados pelo fungo e podem ser uma forma de contágio para esses animais. ${ }^{68}$ Outra medida fundamental seria a distribuição de informações sobre a doença, para profissionais de saúde, veterinários, trabalhadores relacionados com o solo e população no geral. Em um estudo realizado por Martins et al. (2015), ${ }^{69}$ foram promovidas entrevistas acerca da percepção da esporotricose pela população. Dos entrevistados, apenas $20 \%$ possuíam conhecimento sobre a esporotricose e $50 \%$ se automedicaram com produtos de uso tópico. Além disso, os 
entrevistados manifestaram que uma parcela importante dos profissionais de saúde desconhecia a doença, bem como não ofereciam explicações adequadas. Assim, a divulgação de medidas preventivas e de controle da esporotricose, por meio de cartazes, folhetos e outras ações de saúde, conscientizaria a população sobre a doença e como agir.

A a ausência de um programa ou ações de controle da esporotricose em humanos e animais, a falta de unidades de atendimento aos animais e de medicação gratuita para seu tratamento e o desconhecimento das medidas de controle da doença por parte da população, além da dificuldade multifatorial no tratamento da micose nos gatos, são relatados como os principais fatores que contribuem para a ocorrência da epidemia. ${ }^{33,37}$ Com o aumento desenfreado do número de casos da doença, e o lento combate à sua disseminação, a esporotricose tornou-se um grave problema de saúde no Brasil. Sua predominância em populações de baixo poder aquisitivo e o comprometimento de vários determinantes em saúde contribuem para um impacto social preocupante e o agravo das desigualdades sociais. ${ }^{70}$

Nos serviços de saúde, podemos ver esse impacto, principalmente, na atenção primária à saúde, a porta de entrada para pacientes acometidos dos primeiros sinais e sintomas, e na procura por um diagnóstico. Nesse local, a falta de conhecimento pelos profissionais envolvidos, a espera para a realização dos exames e diagnóstico e a ineficaz aquisição do medicamento pelo Sistema Único de Saúde (SUS) tornam o atendimento integral ao paciente oneroso para o governo e desgastante para o paciente. ${ }^{69-72}$ Ainda, o atraso no diagnóstico correto pode contribuir no agravo do quadro da doença, aumentando a morbimortalidade por esporotricose, principalmente em pacientes imunocomprometidos.

Outro fator a ser considerado é a falta de conhecimento da população sobre a esporotricose, que não está diretamente associado ao nível de escolaridade da população, mas, sim, ao fato da insuficiente abordagem da temática nos diversos níveis de ensino da população brasileira, na divulgação de políticas públicas de saúde por parte das autoridades de saúde e nas unidades básicas de saúde. Tal carência de informação eleva o tempo que o paciente procurará um serviço de saúde, a automedicação e o manejo incorreto dos felinos, contribuindo para a perpetuação do ciclo de transmissão. ${ }^{58,69}$ 
No município de Belo Horizonte, devido a um caso de epidemia na região de Barreiro em 2016, os profissionais de saúde receberam treinamento para enfrentar as demandas dos casos de esporotricose humana e animal, compreendendo o fluxo do serviço de vigilância em saúde e notificação de casos no município, além de atuar no fornecimento de educação em saúde para a população. Essa também tem uma função social importante no autocuidado, no manejo do cuidado animal e na conscientização das medidas de prevenção, o que contribui para a coletividade. ${ }^{73}$

Assim, temos que a presença de uma equipe multiprofissional capacitada na atenção primária é fundamental para que o tempo de diagnóstico seja reduzido, a fim de que os prejuízos em saúde no âmbito individual e coletivo sejam minimizados, pois, em muitos casos, o paciente tem transtornos psicossociais, afastamento das atividades de socialização e reclusão da família. ${ }^{70}$ Devido às proporções epidêmicas tomadas pela esporotricose no Brasil, mostra-se fundamental uma mudança nas características sociais, ambientais e comportamentais do governo e da população para extinguir a manutenção do ciclo de transmissão da doença e conseguir o controle dessa epidemia.

\section{CONCLUSÃO}

Em virtude de o fungo habitar o solo, casos de esporotricose sempre existirão. Contudo, em solo brasileiro, a doença adquiriu força em seu caráter zoonótico e apresenta-se em franco crescimento. Relatos obtidos nos últimos anos mostram um aumento exponencial do número de casos de esporotricose, humana e animal. A doença, antes contida no Sudeste e no Sul, agora avança sobre outras regiões e evidencia-se como grave e oneroso problema de saúde, que perdura há mais de uma década.

Pelos dados apresentados, intervenções devem ser realizadas urgentemente, através de políticas públicas de saúde, principalmente no âmbito da atenção primária, a fim de controlar a epidemia brasileira, humana e, sobretudo, animal. Destacam-se as ações para o controle da fonte de infecção, a educação da população, a equipe de saúde e o manejo de animais doentes, que só ajudam a perpetuar de modo eficiente o ciclo da esporotricose.

Torna-se, então, imprescindível o aumento dos investimentos em pesquisa, não 
só para desvendar os determinantes e condicionantes, mas também nortear ações de controle da epidemia. Assim, evidencia-se a necessidade de uma ação conjunta dos serviços de zoonoses e a vigilância epidemiológica nas políticas de saúde a serem criadas, de modo a impactar diretamente as saúdes individual e coletiva.

Agradecimentos: Fundação de Amparo à Pesquisa do Estado de Minas Gerais (FAPEMIG).

\section{REFERÊNCIAS}

1. Arrigala-Moncrieff I, Capilla J, Mayayo E, Marimon R, Marine M, Gene J. Different virulence levels of the species of Sporothrix in a murine model. Clin Microbiol Infect. 2009; 15: 651-55.

2. Ramos-e-Silva M, Vasconcelos C, Carneiro S, Cestari T. Sporotrichosis. Clinics in Dermatology. 2007; 25: 181-87.

3. Macêdo-Sales PA, Souto SRLS, Destefani A, Lucena RP, Machado RLD, Pinto MR et al. Domestic feline contribution in the transmission of Sporothrix in Rio de Janeiro State, Brazil: a comparison between infected and non-infected populations. BMC Vet Res. 2018; 14: 1-10.

4. Madrid IM. Estudo das características fenotípicas, fatores de patogenicidade e suscetibilidade de isolados de Sporothrix schenckii frente a desinfectantes. 2011. 113f. Tese (Doutorado em Veterinária) - Universidade Federal de Pelotas, Pelotas, 2011.

5. Nobre MO, Nascente OS, Meireles MM, Ferreiro L. Drogas antifúngicas para pequenos e grandes animais. Ciênc Rural. 2002; 32: 175-84.

6. Barros MBL, Paes RA, Schubach AO. Sporothrix schenckii and Sporotrichosis. Clin Microbiol Rev. 2011; 24: 633-54.

7. Cordeiro FN, Bruno CB, De Paula, CDR, Mota JAC. Ocorrência familiar de esporotricose zoonótica. An Bras Dermatol. 2011; 86:121-24

8. Fernandes GF, dos Santos PO, Rodrigues AM. Characterization of virulence profile, protein secretion and immunogenicity of different Sporothrix schenckii sensu stricto isolates compared with S. globosa and S. brasiliensis species. Virulence. 2013; 4(3): 241-49.

9. Rodrigues AM, Hoog GS, Pires DDCP, Brilhante RSN, Sidrim JJDC, Gadelha MF, Colombo AL, Camargo ZP. Genetic diversity and antifungal susceptibility profiles in causative agents of sporothrichosis. BMC Infect Dis. 2014; 14:219.

10. Ghosh A, Maity PK, Hemashettar BM, Sharma VK, Chakrabartil. Physiological characters of Sporothrix schenckii isolates. Mycoses. 2012; 45:449-54. 
11. Mendoza M, Hung MB, Díaz AM, Zambrano EA, Díaz E, Albornoz MC. Growth kinetics and morphology of Sporothrix schenckii in diverse culture media. J Mycol Méd. 2005; 15: 127-35.

12. Marimon R, Cano J, Gene J, Sutton DA, Kawasaki M. Sporothrix brasiliensis, S. globosa, and S. Mexicana, three new Sporothrix species of clinical interest. J Clin Microbiol. 2007; 45: 3198-206.

13. Fernandes GF, Santos PO, Amaral CC, Sasaki AA, Godoy-Martinez P, Camargo ZP. Characteristics of 151 Brazilian Sporothrix schenckii isolates from 5 different geographic regions of Brazil: A forgotten and re-emergent pathogen. The Open Mycol J. 2009; 3:48-58.

14. Fernandes GF, Amaral CC, Sasaki AA, Martinez-Godoy P, Camargo ZP. Heterogeneity of proteins expressed by Brazilian Sporothrix schenckii isolates. Med Mycol. 2009; 47: 855-61.

15. Romero-Martinez R, Wheeler M, Guerrero-Plata A, Rico G, Torres-Guerrero H. Biosynthesis and functions of melanin in Sporothrix schenckii. Infect Immun. 2000; 68:3696-703.

16. Almeida-Paes R, Frases S, Monteiro PCF, Gutierrez-Galhardo MC, Zancopé-Oliveira RM, Nosanchuk JN. Growth conditions influence melanization of Brazilian clinical Sporothrix schenckii isolates. Microb Infect. 2009; 11: 554-62.

17. Nobre MO, Antunes TA, Faria RO, Cleff MB, Fernandes CG, Muschner AC et al. Differences in virulence between isolates of feline sporotrichosis. Mycopathol. 2005; 160: 43-49.

18. Kong X, Xiao T, Lin J, Wang Y, Chen HD. Relationships among genotypes, virulence and clinical forms of Sporothrix schenckii infection. Clin Microbiol Infect. 2006; 12: 1077-081.

19. Brito MMS, Conceição-Silva F, Morgado FN, Raibolt PS, Schubach A, Schubach TP et al. Comparison of virulence of different Sporothrix schenckii clinical isolates using experimental murine model. Med Mycol. 2007; 45: 721-29.

20. Marimon R, Serena C, Gené J, Cano J, Guarro J. In vitro antifungal susceptibilities of five species of Sporothrix. Antimicrob Agents Chemother. 2008; 52: 732-34.

21. Sasaki AA, Fernandes GF, Rodrigues AM, Lima FM, Marini MM, Feitosa LDS et al. Chromosomal polymorphism in the Sporothrix schenckii complex. Plos One. 2014; 9:1-13.

22. Chakrabarti A, Bonifaz A, Gutierrez-Galhardo MC, Mochizuki T, Li S. Global epidemiology of sporotrichosis. Med Mycol. 2015; 53: 3-14.

23. Criseo G, Malara G, Romeo O, Puglisi-Guerra A. Lymphocutaneous sporotrichosis in a immunocompetent patient: a case report from extreme southern Italy. Mycopathol. 2008; 166: 159-62.

24. De Oliveira MM, Veríssimo C, Sabino R, Aranha J, Zancopé-Oliveira RM, Sampaio P, Pais C. First autochtone case of sporotrichosis by Sporothrix globosa in Portugal. Diagn Microbiol Infec Dis. 2014; 78:388-90. 
25. Xirotagaros G, Drogari-Apiranthitou M, Tsamakis C, Theotokoglou S, Tofas P, Van Diepeningen et al. Imported lumphocutaneous sporotrichosis in Greece. $\mathrm{Br}$ J Dermatol. 2015; 173: 291-93.

26. Kauffman CA, Bustamante B, Chapman SW, Pappas PG. Clinical Practice Guidelines for the Management of Sporotrichosis: 2007 Update by the Infectious Diseases Society of America. Clin InfectDis. 2007; 45: 1255-265.

27. Sterling JB, Heymann WR. Potassium iodide in dermatology: a $19^{\text {th }}$ century drug for the $21^{\text {st }}$ century uses pharmacology, adverse effects, and contraindications. J Am Acad Dermatol. 2000; 43: 691-97.

28. Rosa CS, Meinerz ARM, Osório LG, Cleff MB, Meireles MCA. Terapêutica da esporotricose: revisão. Sci Anim Health. 2017; 5: 212-228.

29. Larsson CE. Esporotricose. Braz J Vet Res Anim Sci. 2011; 48: 250-59.

30. Mahlberg MJ, Patel R, Rosenman K. Fixed cutaneous Sporotrichosis. Dermatol Online J. 2009; 15:5.

31. Castro VB, Portela C, Oliveira MC, Obadia DL, Gripp AC. Esporotricose e seu polimorfismo clínico: um caso de lesões ulceradas na região centro facial em paciente adulto. Rev SPDV. 2018; 75: 87-90.

32. Cruz LCH. Complexo Sporothrix schenckii. Revisão de parte da literatura e considerações sobre o diagnóstico e a epidemiologia. Vet Zootec. 2013; 20: 8-28.

33. Rodrigues AM, Hoog SD, Camargo ZPD. Emergence of pathogenicity in the Sporothrix schenckii complex. Med Mycol. 2013; 51: 205-12.

34. Rossi CN, Odaguiri J, Larsson CE. Clinical and epidemiological characterization of sporotrichosis in dogs and cats (São Paulo, Brazil). Ciên Agrárias. 2013; 34: 3889896.

35. Fukushiro R. Epidemiology and ecology of sporotrichosis in Japan. Zentralbl Bakteriol Mikrobiol Hyg A. 1984; 257: 228-33.

36. Mochizuki T, Anzawa K. Deep mycosis in Japan. In: Eun HC, Kim SC, Lee WS (eds.). Aging and skin diseases. Special book of the 22nd World Congress of Dermatology 2011; Seoul, Korea: Medrang Inc: 393-397.

37. Barros MBDL, Schubach TP, Coll JO, Gremião ID, Wanke B, Schubach A. Esporotricose: a evolução e os desafios de uma epidemia. Rev Panam Salud Publ. 2010; 27: 455-60.

38. Schubach A, Barros MB, Wanke B. Epidemic sporotrichosis. Curr Opin Infect Dis. 2008; 21: 129-33.

39. Bonifaz A, Araiza J, Perez A, Ochoa LA, Toriello C. Intradermal test with sporotrichin in a community in the Sierra Norte de Puebla. Dermatol Rev Mex. 2013; 57: 428-32.

40. Pappas PG, Tellez I, Deep AE et al. Sporotrichosis in Peru: description of an area of hyperendemicity. Clin Infect Dis. 2000; 30: 65-70. 
41. Barros MB, Schubach Ade O, do Valle AC, et al. Cat-transmitted sporotrichosis epidemic in Rio de Janeiro, Brazil: description of a series of cases. Clin Infect Dis. 2004; 38: 529-35.

42. Vismer HF, Hull PR. Prevalence, epidemiology and geographical distribution of Sporothrix schenckii infections in Gauteng, South Africa. Mycopatho. 1997; 137: 137-43.

43. Bhutia PY, Gurung S, Yegneswaran PP. A case series and review of sporotrichosis in Sikkim. J Infect Develop Countries. 2011; 5:603-08.

44. Helm MAF, Berman C. The clinical, therapeutic and epidemiological features of the sporotrichosis infection on the mines. In: Proceeding of the Transvaal Mine Medical Officers' Association. Sporotrichosis infection on mines of the Witwatersrand. Johannesburg: The Transvaal chamber of mines. 1947: 59-74.

45. Schubach AO, Barros MBL, Schubach TMP, Francesconi-do-Valle AC, GuiterrezGalhardo MC, Sued M et al. Primary conjunctival sporotrichosis two cases from a zoonotic epidemic in Rio de Janeiro, Brazil. Corena J. 2005; 24: 491-493.

46. Conti-Diaz IA. Epidemiology of sporotrichosis in Latin America. Mycopathol. 1989; 108: 113-16.

47. Kano R, Nakamura $Y$, Watanabe $S$, Tsujimoto $H$, Hasegawa A. Identification of Sporothrix schenckii based on sequences of the chitin synthase 1 gene. Mycoses. 2001; 44: 261-5.

48. Vidal G, Rodriguez-de-Kopp N. Sporotrichosis: clinical, therapeutic and epidemiologic overview. Arch Argent Dermatol. 1993; 63: 221-234.

49. Da Rosa AC, Scroferneker ML, Vettorato R, Gervini RL, Vettorato G, Weber A. Epidemiology of sporotrichosis: a study of 304 cases in Brazil. J Am Acad Dermatol. 2005; 52: 451-59.

50. Poester VR, Mattei AS, Madrid IM, Pereira JTB, Klafke GB, Sanchotene KO et al. Sporotrichosis in Southern Brazil, towards na epidemic?. Zoo Publ Health. 2018; 85: 815-821.

51. Gutierrez-Galhardo MC, Freitas DFS, Do Valle ACF, Almeida-Paes R, Oliveira MME, Zancopé-Oliveira RM. Epidemiological Aspects of Sporotrichosis Epidemic in Brazil. Curr Fungal Infect Rep. 2015; 9: 238-45.

52. Silva DT, Menezes RC, Gremião IDF, Schubach TMP, Boechat JS, Pereira AS. Esporotricose zoonótica: procedimentos de biossegurança. Acta Sci Vet. 2012; 40: 1067.

53. Bravo TC. New observations on the epidemiology of sporotrichosis and Sporothrix schenckii complex. Rev Latinoam Patol Clin. 2012; 59: 88-100.

54. Schubach T, Valle A, Gutierrez-Galhardo M, Monteiro P, Reis R, Zancopé-Oliveira $\mathrm{R}$, Marzochi K, Schubach A. Isolation of Sporothrix schenckii from the nails of domestic cats (Felis catus). Med Mycol. 2001; 39: 147-49.

55. Rio de Janeiro. Boletim Epidemiológico Situação Epidemiológica da Esporotricose. Rio de Janeiro: Secretaria de Estado de Saúde do Rio de Janeiro, Gerência de Doenças Transmitidas por Vetores e Zoonoses; 2018; 1. 
56. Schubach TM. Evaluation of an epidemic of sporotrichosis in cats: 347 cases (19982001). J Am Vet Med Assoc. 2004; 224: 1623-629.

57. Almeida AJ, Reis NF, Lourenço CS, Costa NQ, Bernardino MLA, Vieira-Motta O. Esporotricose em felinos domésticos (Felis catus domesticus) em Campos dos Goytacazes, RJ. Braz J Vet Res. 2018; 38: 1438-1443.

58. Rodrigues DKB, Muller EDV, Moraes MCL. Análise do conhecimento sobre zoonoses transmitidas por gatos. Multitemas. 2018; 23: 81-94.

59. Pesquisa Nacional de Saúde 2013. Acesso e utilização dos serviços de saúde, acidentes e violências: Brasil, grandes regiões e unidades da federação IBGE, Coordenação de Trabalho e Rendimento. Rio de Janeiro: IBGE; 2015.

60. Araujo ML, Rodrigues AM, Fernandes GF, Camargo ZP, Hoog GS. Human sporotrichosis beyond the epidemic front reveals classical transmission types in Espírito Santo. Mycoses. 2015; 58: 485-90.

61. Bustamante B, Campos PE. Sporotrichosis: a forgotten disease in the drug research. Expert Rev Anti Infect Ther. 2004; 2: 85-94.

62. Secretaria do Estado de Saúde. Redefine a relação de doenças e agravos de notificação compulsória no âmbito do Estado do Rio de Janeiro. Resolução no 674, de 12 de julho de 2013. Rio de Janeiro, p. 1-8.

63. Informe Técnico 005. Vigilância da esporotricose: orientações sobre vigilância da esporotricose no Estado do Rio de Janeiro. Gerência de Doenças Transmitidas por Vetores e Zoonoses, 2014.

64. Cunha GMCM. Nota Técnica DVIS/CCZ/CIEVS no 4: Orientações sobre notificação da esporotricose no município de Salvador, BA. Secretaria Municipal de Saúde de Salvador, 2018.

65. Brasil. Relação Nacional de Medicamentos Essenciais: Rename 2017/Ministério da Saúde, Secretaria de Ciência, Tecnologia e Insumos Estratégicos, Departamento de Assistência Farmacêutica e Insumos Estratégicos. Brasília: Ministério da Saúde; 2017.

66. Freitas DF, Valle AC, Almeida Paes R, Bastos FI, Galhardo MC. Zoonotic sporotrichosis in Rio de Janeiro, Brasil: a protracted epidemicyet to be curbed. Clin Infect Dis. 2010; 50: 453.

67. Galhardo MC, Oliveira RM, Valle AC, Paes RA, Silvatavares PM, Monzon A et al. Molecular epidemiology and antifungal susceptibility patterns of Sporothrix schenckii isolates from a cat transmitted epidemic of sporotrichosis in Rio de Janeiro, Brazil. Med Mycol. 2008; 46: 141-51.

68. Silva DA, Gremião IDF, Menezes RC, Pereira SA, Figueiredo FB, Ferreira RMC, Pacheco TMV. Micobacteriose cutânea atípica felina no município do Rio de Janeiro - relato de caso. Acta Sci Vet. 2010; 38: 327-31.

69. Martins ACC, Nunes JA, Paheco SJB, Souza CTV. Percepção do risco de transmissão de zoonoses em um centro de referência. Rev Eletr Comun, Info Inov Saúde. 2015; 9: 1-14. 
70. Peter JR, Pires RS, Andrade FC. A esporotricose e seu impacto social. Vittalle - Rev Ciênc Saúde. 2016; 28: 111-114.

71. Silva GM, Howes JC, Leal CAS, Mesquita EP, Pedrosa CM, Oliveira AAF, Silva LBG, Mota RA. Surto de esporotricose felina na região metropolitana do Recife. Braz J Vet Res. 2018; 38: 1767-1771.

72. Orofino-Costa R, Rodrigues AM, Macedo PM, Bernardes-Engemann AR. Sporotrichosis: an update on epidemiology, etiopathogenesis, laboratory and clinical therapeutics. An Bras Dermatol. 2017; 92: 606-620.

73. Moura A, Resende AM, Faria BWF, Emediato CCF, Starling CCD, Santiago CF et al. Esporotricose: Protocolo de enfrentamento da doença em Belo Horizonte. Prefeitura de Belo Horizonte, 2018.

Submissão: outubro de 2017.

Aprovação: fevereiro de 2019. 